\title{
The Role of Modifying Molecular Chains in the Formation of Organized Molecular Films of Organo-modified Inorganic Particles
}

\author{
Manami Iizuka, Yusaku Shidara, and Atsuhiro Fujimori*
}

Graduate School of Sci. and Eng., Saitama University, 225, Shimo-okubo, Sakura-ku, Saitama, 338-8570, Japan

\begin{abstract}
The role of organo-modifying molecular chains in the formation of molecular films of organo-modified nanodiamond is discussed herein based on interfacial chemical particleintegration of organo-modified nanodiamond having a particle size of $5 \mathrm{~nm}$. The surface of nanodiamond is known to be covered with a nano-layer of adsorbed water. This water nano-layer was exploited for organo-modification of nanodiamond with long-chain fatty acids via adsorption, leading to nano-dispersion of nanodiamond in general organic solvents as a mimic of solvency. The organo-modified nanodiamond dispersed "solution" was used as a spreading solution for depositing a mono-"particle" layer on the water surface, and a Langmuir particle layer was integrated at the air/water interface. Multi-"particle" layers were then formed via the Langmuir-Blodgett technique, and were subjected to fine structural analysis. The effect of organo-modification enabled integration and multilayer formation of inorganic nano-particles due to enhancement of the van der Waals interactions between the chains. That is to say, the "encounter" between the organo-modifying chain and the inorganic particles led to solubilization of the inorganic particles and enhanced interactions between the particles, which can be regarded as imparting new function to the organic molecules.
\end{abstract}

\section{Introduction}

Two-dimensional arrangement of nanoparticles has played an important role in the development of modern technology. ${ }^{1}$ Arranged nanoparticles have potential application in numerous technical areas ranging from organic devices to biomaterials. ${ }^{2}$ Furthermore, organic/inorganic hybrid materials ${ }^{3}$ have captured the attention of scientists as well as engineers owing to their remarkably high dimensional stability and gas-barrier performance, in addition to their superior mechanical properties relative to conventional composite materials. ${ }^{4}$

It has been envisioned that two-dimensional arrangement of nanodiamond particles into various materials should effectively enhance several material functionalities. However, it is difficult to obtain regular arrangements of nanodiamond particles because the van der Waals interaction in inorganic materials is relatively weak. To overcome this limitation, surface modification using organic compounds is one prospective means of increasing the affinity between particles. Previously, formation and structure of molecular films comprising organomodified clay are investigated. ${ }^{5}$ In that study, the organo-modified inorganic material formed an extremely condensed monolayer on the surface of water. Moreover, a highly ordered layer structure along the $c$-axis and two-dimensional packing in the $a b$-plane have commonly been constructed by the Langmuir-Blodgett (LB) technique. ${ }^{6}$ In addition, the authors have successfully fabricated highly ordered particle assemblies by the synthesis of longchain fatty acid-modified zirconium oxide (zirconia) by using novel organo-modification methods. ${ }^{7}$

In the present study, high-density and twodimensional arranged nanodiamond organization is performed by an interfacial chemical method. Ultrathin organo-modified nano-diamond films are constructed by floating Langmuir monolayers. The technique proposed in this study is based on the nanodispersion of insoluble inorganic particles by solubilization in a general organic solvent. In general, the outermost layer of the nanodiamond with singlenanometer diameter is covered by a nano-layer of water for structural stabilization. The challenge of organomodification of inorganic particles through the surface nano-phase of water is addressed herein. This nanodispersion of fine inorganic particles can be used as a "spreading solvent" for Langmuir monolayers. In other words, the formation of LB multilayers of nanodiamonds is made possible by using the proposed technique. In this study, LB multilayers of organo-modified nanodiamond films are characterized by out-of-plane and in-plane X-ray diffraction (XRD) and atomic force microscopy (AFM).

\section{Experimental Section}

2.1 Synthesis and characterization of organomodified nanodiamond. A dispersion was prepared by combining an aqueous solution of nanodiamond (detonated nanodiamond, New Metals and Chemicals Co., Ltd., particle size: $4.8 \pm 0.7 \mathrm{~nm}$ ) with a methanolic solution of stearic acid. Toluene was poured into the nanodiamond dispersion with stirring. In this step, the organonanodiamonds migrated from the methanolic dispersion into the toluene phase. Water, methanol, and the remaining unreacted reagent were then removed by rotary evaporation under reduced pressure and decantation. This process changed the surface absorbed hydronium ions (R$\mathrm{H}_{2} \mathrm{O}^{+}$) in the water nano-layer on the particle surface to long-chain alkyl carboxylates, as indicated by IR analyses. It is well known that the nanodiamond surface is coated with a water layer that stabilizes the structure. In this case, it is proposed that carboxylic ions $\left(\mathrm{COO}^{-}\right)$are adsorbed by the hydronium ions on the nanodiamond surface.

2.2 Formation of monolayers on water surface and estimation of molecular arrangement in films.

Corresponding author: fujimori@,fms.saitama-u.ac.jp 
It is possible to achieve uniform size dispersion of the generated organo-nanodiamond on the nanometer scale, where this process can essentially be considered as dissolving organo-nanodiamond in an organic solvent. Therefore, it was also possible to prepare a spreading solvent for formation of a monolayer on the water surface of the nanodiamonds. A Monolayer of organonanodiamond was formed by spreading a toluene solution containing organo-nanodiamond (ca. $1.0 \times 10^{-4} \mathrm{M}$ ) on ultra-pure water $(18.2 \mathrm{M} \Omega \cdot \mathrm{cm})$. The concentration of organo-nanodiamond solutions was estimated by using the average weight of the modified-chain molecules calculated on the basis of the chemical characterization described above. After evaporating the toluene for $30 \mathrm{~min}$, surfacepressure-area $(\pi-\mathrm{A})$ isotherms were recorded at a compression speed of $0.08 \mathrm{~mm} \cdot \mathrm{s}^{-1}$. The temperature of the air/water interface was maintained at a constant value of $15{ }^{\circ} \mathrm{C}$ by circulating thermostatically controlled water around the trough. Measurements of the monolayer properties and LB film transfer were carried out using a USI-3-22 Teflon-coated LB trough (USI Instruments). The monolayers were transferred onto mica (AFM samples) or glass substrates with a ferric stearate monolayer as a hydrophobic underlayer (out-of-plane $\mathrm{XRD}$ and in-plane XRD) at $15^{\circ} \mathrm{C}, 0.2 \mathrm{~mm} \cdot \mathrm{s}^{-1}$, and 25 $\mathrm{mNm}^{-1}$ using the LB method.

\subsection{Surface morphology and particle arrangement in organized films.}

The surface morphology of the transferred monomolecular films was observed using a scanning probe microscope (Dynamic Force Mode, Scan speed : $2.0 \mathrm{~Hz}$, Seiko Instruments, SPA300 with a SPI-3800 probe station) and microfabricated rectangular Si cantilevers with integrated rectangular tips; a spring constant of $1.4 \mathrm{~N} \cdot \mathrm{m}^{-1}$ was applied in this process. The large spacing between the layer structures of the films transferred onto the glass substrates was measured using an out-of-plane X-ray diffractometer (Rigaku, Rint-Ultima III; $\mathrm{CuK \alpha}$ radiation, $40 \mathrm{kV}, 40 \mathrm{~mA}$ ) equipped with a graphite monochromator. The in-plane spacing of the two-dimensional lattice of the films was determined using an X-ray diffractometer with different geometrical arrangements, 9 (Bruker AXS, MXP-BX; $\mathrm{Cu} K \alpha$ radiation, $40 \mathrm{kV}, 40 \mathrm{~mA}$, an instrument especially made to order) that was equipped with a parabolic graded multilayer mirror. X-rays were incident at an angle of $0.2^{\circ}$ and the films were slow-scanned at a speed of $0.05^{\circ} / 80 \mathrm{sec}$.

\section{Results and discussion}

The interfacial monolayer behavior of organo-modified inorganic fine particles is extremely interesting in terms of elucidation of whether the inorganic monolayer is strongly influenced by any of the components. Figure 1(a), (b), (c), and (d) show the $\pi-A$ isotherm of a single particle layer on the water surface of organo-nanodiamond and stearic acid as an organo-modifying agent. Figure 1(a) and (b) show a comparison of the monolayer behavior of organo-modified nanodiamond, stearic acid, and $\mathrm{Cd}$ stearate. The horizontal axes in Fig. 1(a) and (b) respectively indicate the "Area" and "Compression percentage", where the "Area" corresponds to "area/molecule" in the case of
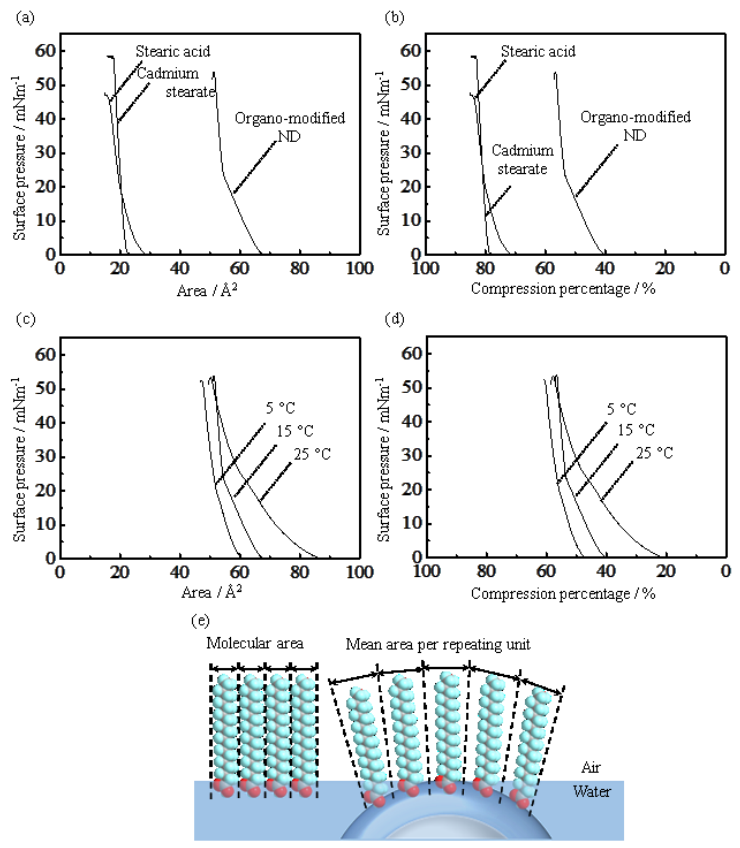

Fig. 1. (a) Surface pressure-area ( $\pi-A)$ and (b) Surface pressure-compression percentage isotherms of monolayers of several materials on the water surface (15 ${ }^{\circ} \mathrm{C}$ ). (c) $\pi$-A, and (d) $\pi$-compression percentage isotherms of single-particle layers of organo-modified nanodiamond on the water surface $\left(5,15\right.$, and $\left.25^{\circ} \mathrm{C}\right)$. (e) Illustration of surface modified agent at air/water interface.

stearic acid and stearic acid cadmium, and indicates the "mean area per hydrophobic repeating unit" in the case of nanodiamond. In this case, the concept of molecular weight cannot be applied because the total number of atoms of nanodiamond particle units cannot be exactly determined. Therefore, considering the surface conformation as shown in Fig. 1(e), the repeating units of only the stearate group of the organo-nanodiamond surface are shown in Fig. 1(a) and (c). However, it is expected that the most accurate comparison would be derived by using the "compression percentage", which indicates the mean of the compression ratio versus the total surface area of the LB trough, as the horizontal axis. Notably, the $\pi$-A curve of organo-modified nanodiamond is clearly different from that of stearic acid and $\mathrm{Cd}$ stearate. From the results of the $\pi$-A isotherms of the organo-modified ND monolayer, both expanded and condensed phases were confirmed in the low and high surface pressure regions, respectively. In addition, two-dimensional phase transition from the expanded phase to the condensed phase is indicated by the changes in the inclination of $\pi$-A curve of organonanodiamond monolayer. The appearance of this phase transition is an effect derived from the organic component, while the appearance of a clear crystalline phase in the high-pressure region is believed to be an effect of the inorganic component. The temperature dependence of the $\pi$-A curve was evaluated to confirm the occurrence of the effect derived from the organic component (Fig. 1(c) and (d)). Specific condensation and expansion behavior typical of the $\pi$-A isotherms of organic compounds was confirmed with the change of the subphase temperature. 

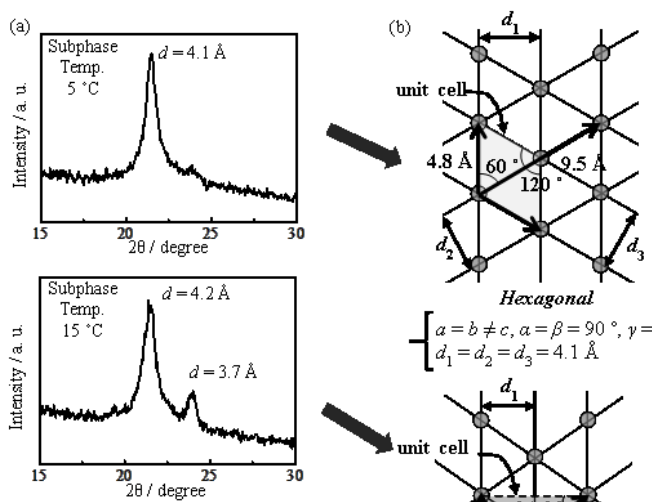

$\left\{\begin{array}{l}a=b \neq c, a=\beta=90^{\circ}, y=120^{\circ} \\ d_{1}=d_{2}=d_{3}=4.1 \AA\end{array}\right.$
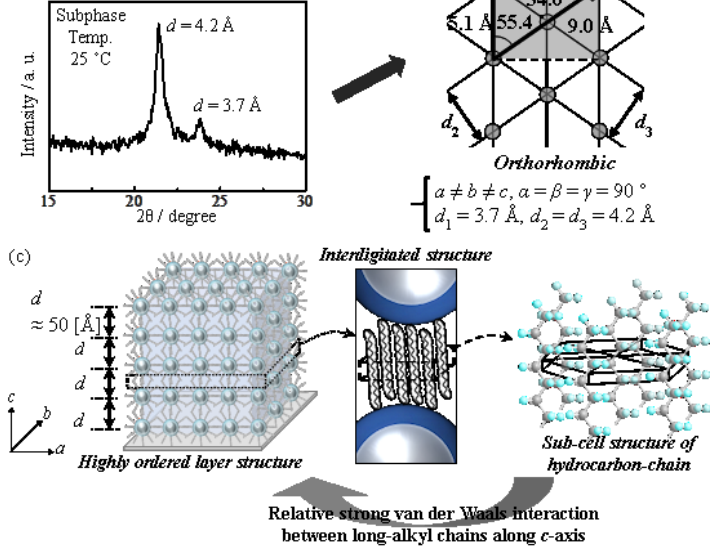

Fig. 2. (a) Mesoscopic AFM images of Z-type singleparticle layer of organo-modified nanodiamond transferred by LB method $\left(5,15\right.$, and $\left.25^{\circ} \mathrm{C}\right)$. (b) Out-ofplane XRD profiles of multi-particle layers of organomodified nanodiamond transferred by LB method $(5,15$, and $25^{\circ} \mathrm{C}$ ). (c) Schematic illustration of multi-particle layered organization of organo-modified nanodiamond. (d) Photograph of the occurrence behavior of structural colors of step-wise multilayers of organo-modified nanodiamond.

This single-particle layer on the water surface was transferred to a mica substrate at various temperatures by using the upstroke LB method. Figure 2(a) shows AFM images of these Z-type single-particle layers. Formation of an organized molecular film of organo-modified nanodiamond was also confirmed by Laser Raman spectroscopy. A diamond-specific $1330 \mathrm{~cm}^{-1}$ Raman shift band ${ }^{10}$ was confirmed in the Raman spectrum of the organized film, in addition to the C-C band at $1098 \mathrm{~cm}^{-1}$. In a nutshell, it was not possible to obtain a quite homogeneous single particle film at this stage. In particular, because the height is about three times the particle diameter in the case of the layer transferred at 5 ${ }^{\circ} \mathrm{C}$, it seems that there are sites where the particles aggregate and pile up along the height direction. In the case of the single-particle layers transferred at 15 and 25 ${ }^{\circ} \mathrm{C}$, although the height direction is homogeneous and consistent with single particle size, the tendency towards particle integration in the in-plane direction is not necessarily high. This problem is eliminated by the "repeating compression-expansion method" discussed in an ensuing section.

On the other hand, the formation of a quite highly ordered layer arrangement along the height direction was inferable
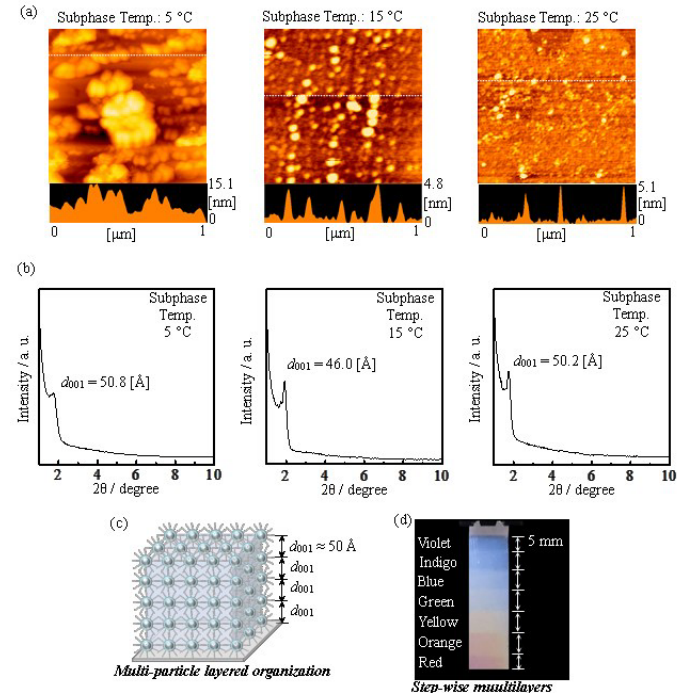

Fig. 3. (a) In-plane XRD profiles of multi-particle layers of organo-modified nanodiamond transferred by LB method $\left(5,15\right.$, and $\left.25^{\circ} \mathrm{C}\right)$. (b) Schematic models of twodimensional lattices formed by long hydrocarbons on organo-modified nanodiamond surface of their LB films transferred at several subphase temperature. Schematic illustration of formation of sub-cell structure in high-order layered organization of organo-modified nanodiamond based on the contribution of strong van der Waals interaction between alkyl chains.

from the data in Fig. 2(b). The results of out-of-plane Xray diffraction of the $\mathrm{LB}$ multilayers indicates that an organized film was transferred at all subphase temperatures, and the films showed a clear long-period value at around $5 \mathrm{~nm}$. This value corresponds to the particle lamination period as shown in Fig. 2(c). The diffraction peaks in transferred at $15{ }^{\circ} \mathrm{C}$ and transferred at $25{ }^{\circ} \mathrm{C}$ were comparatively sharper than that in transferred at $5{ }^{\circ} \mathrm{C}$; therefore, more advanced layered structures were formed in the former films. As further evidence, the multilayers fabricated stepwise at $15^{\circ} \mathrm{C}$ exhibited a clear structural related color up to wavelengths in the visible light region in response to stacking (Fig. 2 (d)). In this case, gradation of structural color has been indicated on the stepwise-multilayers. The violet, indigo, blue, green, yellow, orange, and red colors correspond to 38, 44, 49, 54, 60,65 , and 70 layers of organo-nanodiamond, respectively. Figure 3 shows the in-plane X-ray diffraction profiles of multi-particle layers of organo-nanodiamond that were transferred at several temperatures. Unfortunately, the diffraction peak in the low angle region corresponding to the long spacing in the out-of plane XRD pattern was not observed. Based on the AFM results, this observation may be reasonable. The arrangement of the particles in the twodimensional plane would not be highly regular at this stage. However, the modifying long-chain alkyl group was found to have a high degree of order in the particle layers. A single peak corresponding to the isotropic hexagonal (100) plane was apparent at $4.1 \AA$ in the case of the multi-particle layers fabricated at the subphase temperature of $5{ }^{\circ} \mathrm{C}$. Additionally, the appearance of double peaks in the case 


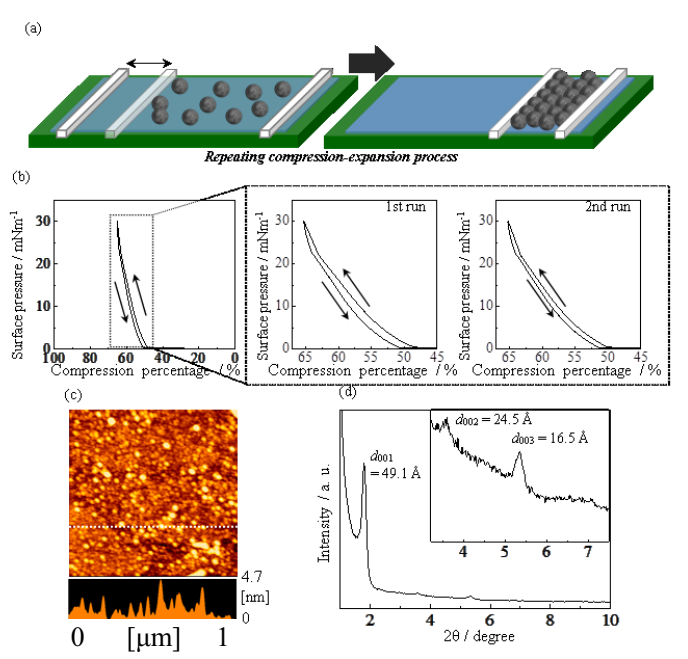

Fig. 4. (a) Schematic illustration of "repeating compression-expansion method". (b) Hysteresis curves of $\pi$-A isotherms of single-particle layers of organomodified nanodiamond on the water surface $\left(15^{\circ} \mathrm{C}\right)$. (c) AFM image of single-particle layer of organo-modified nanodiamond fabricated by "repeating compressionexpansion method" at 6 times. (d) Out-of-plane XRD profiles of multi-particle layers of organo-modified nanodiamond performed "repeating compressionexpansion method" at 6 times.

of the multi-particle layers fabricated at the subphase temperatures of 15 and $25{ }^{\circ} \mathrm{C}$ indicates that the modified alkyl chains form a slightly anisotropic orthorhombic system. In view of the ratio of surface modification of the particles by stearic acid, the possibility that the long alkyl chain is densely packed on one particle surface is extremely low. Accordingly, packing of the alkyl chains is expected to generate a two-dimensional crystal array of the alkyl chains between individual particles. In other words, the two-dimensional integration and formation of the layer structure of organo-nanodiamond can be determined, indicating that crystal formation arises from interaction between modified alkyl chains. This inferred model is represented in Fig. 3(c). Sub-cell ${ }^{11}$ formation by the hydrocarbons between the particles is considered to be the origin of the nano-particle integrated organization in this system.

As the next step, the formation of the high-density and low-defect particle layer was addressed. Figure 4(a) shows a schematic illustration of the "repeating compression-expansion process" for generation of the single particle layer on the water surface. In a system where the size of the components is large and the interaction between constituent units is relatively weak, a low-defect layer can be effectively formed from this process. The fact that this technique works effectively reflects the monolayer properties of the inorganic compound. In the case of an organo-modified montmorillonite single particle layer, this technique was effectively applied to fabricate low-defect films. Figure 4(b) illustrates the reversibility and hysteresis of the $\pi$-A isotherm at $15{ }^{\circ} \mathrm{C}$. At each step of the repeating process, the difference in the hysteresis during compressionexpansion becomes less. When this process is performed 6 times, the $5^{\text {th }}$ and $6^{\text {th }}$ curves of the hysteresis loop almost coincide. Therefore, considering that the highest density films had successfully been attained, the resulting monolayer and LB multilayers transferred to the solid substrate were subjected to AFM observation and out-of plane X-ray diffraction. The results confirm a very lowdefect surface morphology (Fig. 4(c)). In addition, the $d_{001}$ peak in the out-of plane X-ray diffraction pattern became very sharp (Fig. 4(d)). Comparing the magnitude of the $D_{001}$ for the crystallites of the fabricated films based on the full width at half maximum before and after performing the sequence of the "repeating compression-expansion process" for six cycles, the diameter of the crystallites clearly increased from $364 \AA$ to $461 \AA$ (the Scherrer constant $K=0.94$ was used).

\section{Conclusions}

The role of organic chain molecules used for surface modification of inorganic particles was discussed via construction of two-dimensional integration and layered lamination of organo-modified nanodiamond. Multiparticle layered films of organo-nanodiamond were constructed by using the LB technique. Mesoscopic-scale AFM images of this single-particle layer of organonanodiamond fabricated by the "repeating compressionexpansion process" demonstrated formation of a highdensity surface with a uniform height of $5 \mathrm{~nm}$. These regular structures are based on the strong van der Waals interactions of the organo-modifying chains.

\section{References}

1. Y. Yin, Y. Lu, B. Gates, Y. Xia, J. Am. Chem. Soc., 123, 8718 (2001)

2. A. Fujimori, N. Sato, S. Chiba, Y. Abe, Y. Shibasaki, J. Phys. Chem. B, 114, 1822 (2010)

3. K.M. Kim, D.K. Keum, Y. Chujo, Macromolecules, 36, 867 (2003)

4. A. Fujimori, N. Ninomiya, T. Masuko, Polym. Eng. Sci., 48, 1103 (2008)

5. A. Fujimori, J. Kusaka, R. Nomura, Polym. Eng. Sci., 51, 1099 (2011)

6. T. Yamamoto, Y. Umemura, O. Sato, Y. Einaga, J. Am. Chem. Soc., 127, 16065 (2005)

7. A. Fujimori, N. Honda, H. Iwashita, Y. Kaneko, S. Arai, M. Sumita, S. Akasaka, Colloids Surf. A, 446, 109 (2014)

8. A. Fujimori, Y. Sugita, H. Nakahara, E. Ito, M. Hara, N. Matsuie, K. Kanai, Y. Ouchi, K. Seki, Chem. Phys. Lett., 387, 345 (2004)

9. A. Fujimori, T. Araki, H. Nakahara, E. Ito, M. Hara, H. Ishii, Y. Ouchi, K. Seki, Chem. Phys. Lett., 349, 6 (2001)

10. O.A. Shenderova, I.I. Vlasov, S. Turner, G.V. Tendeloo, S.B. Orlinskii, A.A. Shiryaev, A.A. Khomich, S.N. Sulyanov, F. Jelezko, J. Wrachtrup, J. Phys. Chem. C, 115, 14014 (2011)

11. V. Vand, I.P. Bell, Acta. Cryst., 4, 465 (1951) 